\title{
Does Botulinum Toxin A Treatment Enhance the Walking Pattern in Idiopathic Toe-Walking?
}

\author{
Heli Sätilä1,2,3 Anneli Beilmann ${ }^{4}$ Päivi Olsén ${ }^{5}$ Heli Helander $^{5}$ Mari Eskelinen ${ }^{6}$ Heini Huhtala ${ }^{7}$
}

1 Department of Pediatric Neurology, Päijät-Häme Central Hospital, Lahti, Finland

2 Department of Pediatric Neurology, Kanta-Häme Central Hospital, Hämeenlinna, Finland

${ }^{3}$ Department of Pediatric Neurology, Tampere University Hospital, Tampere, Finland

${ }^{4}$ Department of Pediatric Neurology, Lappeenranta Central Hospital, Lappeenranta, Finland

5 PEDEGO Research Unit (Research Unit for Pediatrics, Dermatology, Clinical Genetics, Obstetrics and Gynecology), Oulu University Hospital and University of Oulu, Oulu, Finland

6 Physiatric Department, Päijät-Häme Central Hospital, Lahti, Finland

${ }^{7}$ School of Health Sciences, University of Tampere, Tampere, Finland

\author{
Address for correspondence Heli Sätilä, MD, PhD, Department of \\ Pediatric Neurology, Päijät-Häme Central Hospital, \\ Keskussairaalankatu 7, Lahti 15850, Finland \\ (e-mail: heli.satila@phsotey.fi).
}

Neuropediatrics 2016;47:162-168.

\begin{abstract}
Objective We conducted a randomized controlled trial to evaluate whether a combination of repeated botulinum toxin A (BTX-A) and conservative treatment is more effective in decreasing toe-walking than conservative treatment alone at 24 months follow-up.

Patients and Methods Children between 2 and 9 years of age were randomized either into the conservative (CO) or botulinum treatment (BTX) group. The treatment in the CO group consisted of firm shoes, night splints, a home stretching program and physiotherapy. The BTX arm had all the same conservative treatments added with calf muscle BTX-A injections repeated in 6 months intervals if needed. Change in toe-walking pattern, ankle range of movement (ROM), and overall function were assessed at baseline and $6,12,18$, and 24 months posttreatment.

Results A total of 30 toe-walkers participated: 14 in $\mathrm{CO}$ and 16 in BTX group. At 24 months, all children in the BTX group and $85 \%$ in the CO group evaluated by the

\section{Keywords}

- idiopathic toewalking

- toe-walking

- botulinum toxin $\mathrm{A}$

- conservative treatment blinded physiotherapist ( $p=0.065$ ), 75\% in the BTX group and 70\% in the CO group graded by the research physiotherapist $(p=0.730)$, and $50 \%$ in the BTX group and $54 \%$ in the CO group reported by the parents ceased toe-walking $(p=0.837)$. The most prominent change was noted during the 1st year. The BTX group seemed to reach the goal earlier. No significant differences between the treatment groups in function or in ankle ROM ensued.

Conclusion Adding BTX injections did not significantly enhance the goal to walk either flat foot or with heel strike at 24 months posttreatment.
\end{abstract}

received

December 5, 2015

accepted after revision

February 15, 2016

published online

April 18, 2016 (c) 2016 Georg Thieme Verlag KG

Stuttgart · New York
Dol http://dx.doi.org/ 10.1055/s-0036-1582138. ISSN 0174-304X. 


\section{Introduction}

Toe-walking, a pattern of walking with toe-toe gait, is considered a part of normal development of the gait. However, it is abnormal if persisting over the age of 2 years. ${ }^{1}$ Toewalking may be caused by congenital short Achilles tendon, neuropathic or myopathic disorders, cerebral palsy, and spinal dysraphism, or be associated with developmental or neuropsychiatric disorders. ${ }^{2}$ Toe-walking is called habitual or idiopathic (ITW) in the absence of any known cause. With an estimated prevalence of 5 to $12 \%$ in childhood, ITW is a common reason for referral to a pediatric neurologist, orthopedist, or physiatrist. ${ }^{3,4}$ If persistent at school-age, muscle or joint pain, ankle joint deformities, shortening of the triceps surae muscle-Achilles tendon complex, and decreasing of passive ankle joint range of movement (ROM) may ensue with inability to participate in sports or being bullied by peers. ${ }^{2,4}$

The treatment options include observation, conservative methods (orthotics, special shoes, splinting, calf muscle stretching, serial casting), and soft-tissue surgery (lengthening of the triceps surae muscle-tendon complex). ${ }^{5}$ Recently, a less invasive treatment, injecting botulinum toxin A (BTX-A) into the calf muscles either alone or combined with serial casting, has become commonplace. ${ }^{6-10}$

Few studies have reported on the efficacy and safety of BTX-A treatment in ITW. ${ }^{6-9}$ In these nonrandomized, open studies without control group toe-walkers aged 2 to 18 years were injected with 3 to $6 \mathrm{U} / \mathrm{kg}$ of BTX-A per leg, followed by 1 to 3 weeks serial casting, bracing, and/or physiotherapy. Within a follow-up period varying from 2 to 30 months, 27 to $100 \%$ of participants were reported to stop toe-walking. Some patients required a repeated treatment at 3 to 12 months, but mostly single injection treatment was performed. Few children went on to surgery. A randomized study comparing single BTX-A treatment with BTX-A coupled with serial casting found that adding BTX-A did not improve the results compared with casting alone. ${ }^{10}$

To our knowledge, no previous study has compared BTX-A with conservative treatment. We conducted a randomized controlled trial to evaluate the hypothesis that a combination of conservative treatment (night splints, firm shoes, physiotherapy, and home stretch program) and repeated BTX-A injections are more effective in decreasing toe-walking than conservative treatment alone at 24 months follow-up.

\section{Patients and Methods}

\section{Patients}

Patients were recruited from five pediatric neurology departments treating ITW. Children were considered for inclusion if they had toe-walked at least 6 months and if the pattern fulfilled grades 1, 2, 3, or 4 on the toe-walking severity scale (TWSS, - Table 1) ${ }^{11}$ at baseline. The children had to be 2 to 10 years of age with no other explanation for the toe-walking (cerebral palsy, neuropathy, myopathy, autism, developmental disorders, short tendon Achilles), and to have passive ankle dorsiflexion with extended knee 0 degrees or more
Table 1 Toe-walking severity scale

\begin{tabular}{|l|}
\hline Grade 1: Toe-walking $76-100 \%$ of time \\
\hline Grade 2: Toe-walking $51-75 \%$ of time \\
\hline Grade 3: Toe-walking $26-50 \%$ of time \\
\hline Grade 4 : Toe-walking $10-25 \%$ of time \\
\hline $\begin{array}{l}\text { Grade } 5: \text { Walking plantigrade but heel off early } \\
\text { (occasional toe-walking }<10 \% \text { accepted) }\end{array}$ \\
\hline Grade $6:$ Walking with normal heel strike \\
\hline
\end{tabular}

Source: Adapted from ICF-CY, WHO $2001 .^{11}$

(no contracture). Exclusion criteria were previous treatment with serial casting or botulinum toxin or surgery. A research pediatric neurologist at each hospital (H. S., A. B., P. O., H. H.) diagnosed and evaluated all children. The study was approved by the local research ethics committees and the European Medicines Agency.

The family was given both written and oral information about the study. After the baseline measurements, informed written consent was obtained from caregivers. Patients meeting the study criteria were randomized either into the conservative (CO) or botulinum treatment (BTX) group. Randomization was done with the use of sealed envelopes prepared by a statistician, with randomly permuted blocks of four. Younger children (2-6 years) versus older (7-10 years) children were enrolled in separate categories and then paired on toe-walking severity measured by the TWSS. The grade given by the research physiotherapist at baseline assessment was taken into account in the randomization. The goal for the treatment was set on grade 5 . Grade 6 was considered as a goal better than expected.

\section{Intervention}

The treatment in the CO group consisted of indoor shoes with a firm heel cup and straps to be worn daily at daycare or school, night splints made of soft cast to be worn at least 5 nights a week, and a home stretching program performed 5 days a week, for a minimum of 10 minutes/day incorporated into the family's daily schedule (for instance performed during children's TV-programs or while watching videos). The instructions for the home program were given both orally and in writing by the research physiotherapist. The written instructions included photos of the stretching movements, and the parents and child could choose two out of six options for a day. If the child had a sporty hobby, he/she was encouraged to attach the stretching to that. Also, regular physiotherapy was started once a week, lasting for the first study year to supervise and encourage continuing the stretching. The BTX arm had all the same conservative treatments added with gastrocnemius-soleus complex BTX-A injections repeated in 6 months intervals if needed (TWSS grades 1-4, need for the repeat injection determined at 6,12 , and 18 months assessment). In the CO group, if the toewalking pattern was negatively affecting daily life (TWSS grades 1-4) and the caregivers insisted on a BTX injection, the child could be injected after 6 months follow-up. 
The BTX-A injections were given into both legs with electromyogram-neurostimulation amplifier guidance, one injection into the proximal part of both heads of the gastrocnemius and one more distally into soleus, three injections in each calf. All injections were given by the research physician at a constant dose of $3 \mathrm{U} / \mathrm{kg}$ per head of the gastrocnemius muscle and $2 \mathrm{U} / \mathrm{kg}$ into soleus ( $100 \mathrm{U} / \mathrm{mL} 0.9 \%$ saline, BOTOX, Allergan Inc., Irvine, CA), making a total dose of $16 \mathrm{U} / \mathrm{kg}$. Doses over $50 \mathrm{U}(0.5 \mathrm{~mL})$ were divided into two adjacent sites. Local lidocaine cream and light conscious sedation with midazolam (0.3 $\mathrm{mg} / \mathrm{kg}$, maximum $10 \mathrm{mg}$ per child) was used. After injection, flexing-extending movement was performed to enhance the spreading of BTX-A in the muscle bulk. Treatment was given on an outpatient basis.

\section{Clinical Assessments}

Assessments were made at baseline and 6, 12, 18, and 24 months posttreatment by the same pediatric research physicians and physiotherapists, who continued with the child throughout the research period. In the $\mathrm{CO}$ arm, one clinical assessment was performed at 3 months to ascertain that the family had understood the instructions and everything was going fine. In the BTX arm (and in the CO arm if injected), there was always a posttreatment assessment 1 month after each given injection. Because of the study design the researchers or parents were not blinded to the treatment arms.

\section{Outcome Measures on Impairment and Function}

Change in severity of toe-walking pattern by TWSS was set as the primary outcome. Active and passive ankle ROM and parental questionnaire of the lower extremity function assessment test ${ }^{7}$ were secondary outcomes. At each assessment, popliteal angle of knee and ankle ROM with the knee extended and flexed was measured by manual goniometry. The walking and playing of the child were video recorded for 15 minutes. Research physiotherapist scored the severity of the toe-walking pattern by TWSS. This was done by estimating the time, which the child spent on his/her toes during the 60-minute assessment.

Also, the parents were asked to score the severity of the toewalking pattern by the TWSS, estimating the time their child walked on toes at home while not being at the kindergarten or school. Likewise, the parents filled a questionnaire of the lower extremity function assessment test at each appointment. An open questionnaire asked them to report the adverse effects of the BTX-A treatment. The parents classified the adverse effects as "severe," "moderate," or "mild."

After cessation of the study, a pediatric physiotherapist (M. E.) not involved with the measurements and blinded to the treatment groups or time sequences scored each participant from 15 minutes compiled video recordings with the TWSS. This score was taken into account together with the researcher's and parents' scorings when analyzing and reporting the results.

\section{Statistical Analysis}

Statistical analysis was based on the intention-to-treat principle. Sample size calculation was based on the primary endpoint of group differences in TWSS at 24 months. Grades
5 and 6 were considered as successful. In the CO group, 30\% and in the BTX group, 90\% were expected to reach the goal. With $80 \%$ power and 0.05 type I error 12 patients were needed in both groups to be able to reject the null hypothesis that the success rates are equal. Student $t$-test was performed for comparison of continuous variables between groups and Fisher exact tests for discrete variables. The changes of skew continuous and ordinal data within groups were tested using Friedman test. The $p$ values of $\leq 0.05$ were considered statistically significant. All statistical analyses were performed using SPSS Statistics for Windows version 22.0.

\section{Results}

\section{Participants}

Overall, 30 toe-walkers (18 males and 12 females) between the ages of 2 and 9 years participated. A positive family history of toe-walking was reported in 57\% of the children. Most of the children were born with no complications (93\%), three children (10\%) were born preterm, and six (20\%) by cesarean section. Chief complaints beside toe-walking were clumsiness in 11 (37\%), leg pain in 16 (53\%), fatigue in 17 (57\%), hyperactivity in 13 (43\%), speech delay in 5 (17\%), difficulties in fine motor functions in $9(30 \%)$, and slight ankle valgus deformity in 9 (30\%). Eleven (37\%) started independent walking under the age of 12 months, the rest between 12 and 18 months. None had started walking after 18 months. A total of 13 (43\%) walked on tiptoe as soon as they began independent walking. On entering the study, 23 (78\%) were toewalking even with shoes on and 6 were reported by their parents not being able to walk at all on heel-contact pattern.

\section{Treatment Groups}

A total of 14 were randomized into the $\mathrm{CO}$ group and 16 into the BTX group. One patient in the CO group was lost to follow-up after 12 months. At baseline, there were no between-group differences (-Table 2). In the BTX group, all 16 patients received BTX-A treatment at baseline, 8 received a second treatment at 6 months and 1 received a third treatment at 12 months after which no one needed further injections. In the CO group, three wanted BTX-A treatment: one received treatment at 12 months, one at 6 and 12 months, and one at 12 and 18 months. All children wore night splints and firm shoes regularly except one in the $\mathrm{CO}$ group.

\section{Severity of the Toe-Walking Pattern by the Toe- Walking Severity Scale}

Both groups improved significantly during the 24-month follow-up (-Fig. 1). The most prominent change in toewalking pattern was noted during the 1 st year after which there was a plateauing. The BTX group seemed to reach the goal earlier. Of the 29 patients who were followed up for 24 months, all 16 in the BTX group (100\%) and 11/13 (85\%) in the CO group ceased toe-walking (grades 5 or 6 ) when evaluated by the blinded physiotherapist, the between-group difference being nearly significant ( $p=0.065)$. Twelve (75\%) in the BTX group and $9(70 \%)$ in the CO group graded by the research physiotherapist, and 8 (50\%) in the BTX group and 7 
Table 2 Patient characteristics at baseline

\begin{tabular}{|c|c|c|}
\hline & Conservative & Botulinum toxin $\mathrm{A}$ \\
\hline Patients & 14 & 16 \\
\hline Age $(y)$ (mean [range]) & $4.9(2.0-9.0)$ & $5.3(2.5-8.0)$ \\
\hline Sex (male/female) & $9 / 5$ & $9 / 7$ \\
\hline Use of night splints before study (yes/no) & $6 / 8$ & $7 / 9$ \\
\hline Use of shoes before study (yes/no) & $2 / 12$ & $3 / 13$ \\
\hline Birth weight $(1,500-2,500 />2,500 \mathrm{~g})$ & $0 / 14$ & $1 / 15$ \\
\hline Independent walking (< $12 \mathrm{mo} / 12-18 \mathrm{mo})$ & $6 / 8$ & $5 / 11$ \\
\hline \multicolumn{3}{|l|}{ Severity of toe-walking pattern at start } \\
\hline Grade 4 & 1 & 2 \\
\hline Grade 3 & 4 & 4 \\
\hline Grade 2 & 4 & 3 \\
\hline Grade 1 & 5 & 7 \\
\hline \multicolumn{3}{|l|}{ Lower extremity function scores } \\
\hline Percentage (mean, range) & $76(60-95)$ & $71(30-95)$ \\
\hline \multicolumn{3}{|l|}{ Ankle ROM (degrees) (mean [SD]) } \\
\hline Active knee extended (right) & $2.3(6.7)$ & $3.5(5.9)$ \\
\hline Active knee extended (left) & $2.8(7.7)$ & $2.5(9.1)$ \\
\hline Active knee flexed (right) & $9.8(7.3)$ & $9.3(7.0)$ \\
\hline Active knee flexed (left) & $6.6(8.9)$ & $9.9(9.2)$ \\
\hline Passive knee extended (right) & $11.1(7.8)$ & $11.5(7.2)$ \\
\hline Passive knee extended (left) & $13.4(7.8)$ & $10.8(7.1)$ \\
\hline Passive knee flexed (right) & $16.0(7.7)$ & $17.6(6.4)$ \\
\hline Passive knee flexed (left) & $17.7(7.5)$ & $16.6(7.3)$ \\
\hline
\end{tabular}

Abbreviations: ROM, range of movement; SD, standard deviation.

(54\%) in the CO group reported by the parents ceased toewalking, the between-group differences not being significant ( $p=0.730$ and 0.837 , respectively). Analyzing the data by three groups (CO, one BTX-A treatment, and 2-3 BTX-A treatments) did not change these results.

According to blinded physiotherapist's evaluation, all three in the CO group receiving BTX-A treatment attained the goal at 24 months. However, parents perceived these children to benefit from the treatment, but still walk on their toes 25 to $50 \%$ of the time at home (grades 3, 4, and 4). In total, only one patient was doomed to remain unchanged throughout the study period by all three evaluating parties, one patient by the research physiotherapist only, and one by the parents only (the child followed up for 12 months). These children were all in the $\mathrm{CO}$ group.

\section{Ankle Dorsiflexion}

At pretreatment, all children had normal knee and ankle ROM (no contracture). During the study period, the median change from baseline in passive ankle ROM both knee extended and flexed declined statistically significantly in both legs in the CO group $(p<0.05)$ and in right leg knee extended in the BTX group ( $p<0.05$ ). At 24 month end point, passive ankle ROM had declined in 10 (CO: 5; BTX: 5), remained the same in 4
(CO: 2; BTX: 2), and increased in 15 children (CO: 6; BTX: 9). No significant differences in median change from baseline in ankle ROM between the treatment groups were noted. Due to difficulties in cooperation the active ROM could not be measured in two children.

\section{The Lower Extremity Function Assessment Test}

At baseline, the degree of function was considered good (CO median 75\%, range 60-95\%; BTX median 75\%, range 30-95\%). Still, there was improvement during the 24-month follow-up. In both groups, a significant increase in function was noted during the first 6 months $(p=0.002)$. No significant between-group differences ensued (-Fig. 2).

\section{Adverse Events}

A total of 30 BTX-A treatments were given during the 2 years follow-up, of which 8 (27\%) were without any negative events. Parents reported a total of 38 adverse events: tenderness in the calf ( $n=13)$, clumsiness $(n=9)$, fatigue $(n=5)$, muscle weakness $(n=3)$, irritability $(n=3)$, flu-like symptoms $(n=1)$, loss of appetite $(n=1)$, constipation $(n=1)$, rash $(n=1)$, and urgency to urinate $(n=1)$. Adverse events were mostly mild or moderate (87\%). Three were considered severe (i.e., lasting longer than 5 days) by the parents: 

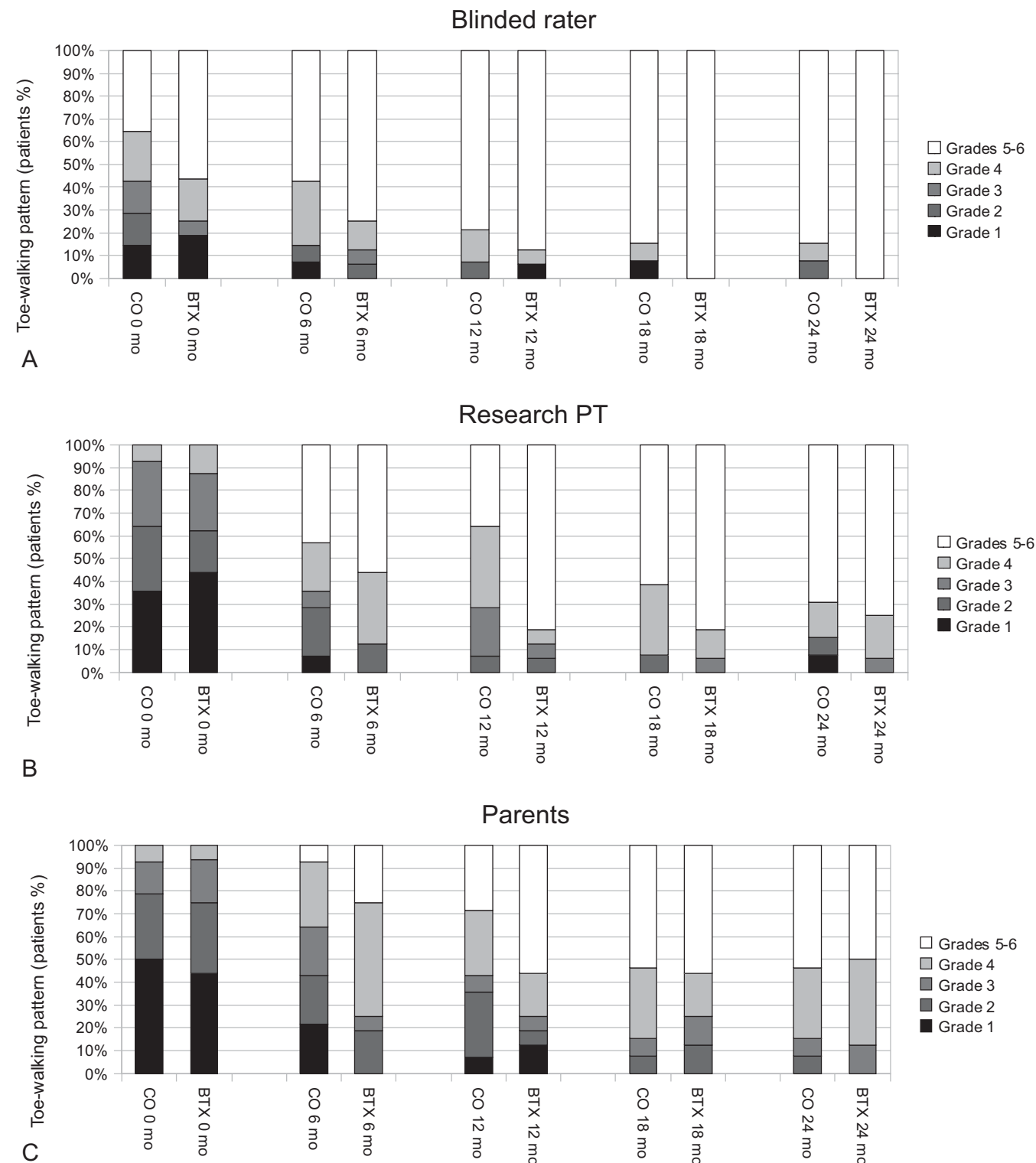

Fig. 1 Outcome of the severity of the toe-walking pattern by the toe-walking severity scale (TWSS) (a) by the blinded physiotherapist, (b) by the research physiotherapist, and (c) by the parents.

clumsiness $(n=1)$, local tenderness $(n=1)$, and constipation $(n=1)$. No one withdrew because of these symptoms. None complained of sore skin or rash while wearing the night splints.

\section{Discussion}

This randomized controlled trial sought to evaluate the hypothesis that a combination of conservative treatment (night splints, firm shoes, physiotherapy, and home stretch program) and repeated BTX-A injections are more effective in decreasing toe-walking pattern than conservative treatment alone at 24 months follow-up. Our results did not support this hypothesis. Both groups improved significantly in terms of children attaining the goal to walk either flat foot or with heel strike, and the between-group difference was nonsignificant. The most prominent change in toe-walking pattern was noted during the first 12 months after the introduction of the treatment regimens. The BTX group seemed to reach the goal earlier.

These response rates concur with those reported in the literature. ${ }^{6-10}$ Compared with the previous studies, our patients were younger, the BTX-A treatments were more systematically repeated, the BTX-A doses were higher, and we used blinded rating along with the parental and researchers' ratings. Night splints were used instead of posttreatment casting.

A child with ITW may spontaneously walk both on a toetoe and a heel-contact pattern, even in the same gait analysis or observation situation. We noticed that the children were less on toes when knowing of being observed (15-minute video taping) than when concentrating in free playing situations, forgetting the surroundings and observers (during the 1 hour playing session). Thus, it can be questioned whether the observational gait analysis with TWSS and rating by a blinded rater represents the child's most common 


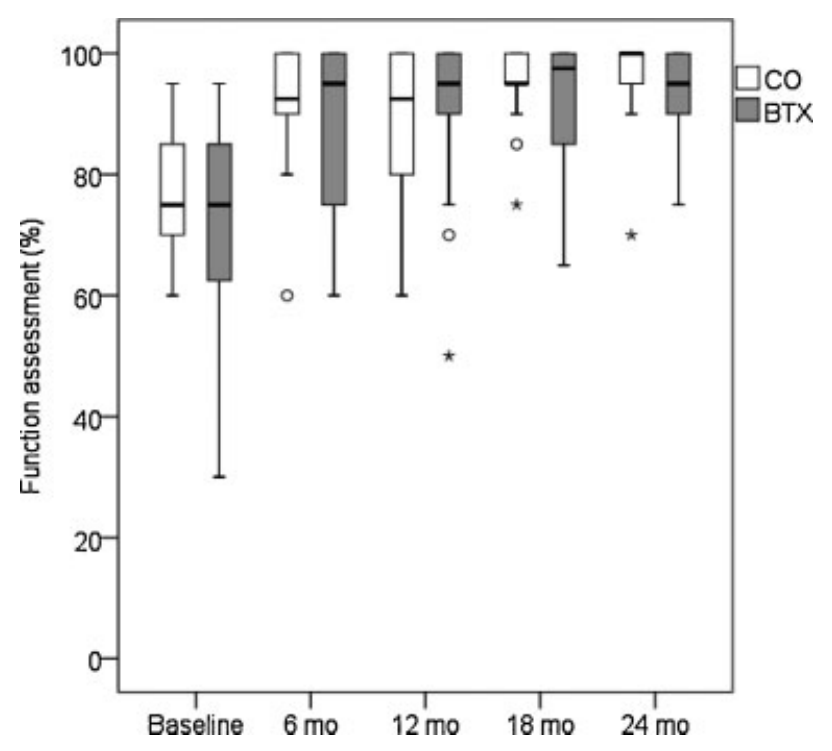

Fig. 2 Boxplots illustrating results for the lower extremity function assessment test at baseline, 6, 12, 18, and 24 months. In y-axis, result points as percentage (\%) of the maximum.

walking performance. The parental reports may give a better indication of the child's situation in normal environment. In our study, the parents seemed to be more critical about their child's performance as they were having in mind the performance at home and leisure time. This was also what they were instructed to rate. The researchers evaluated the 1-hour and the blinded rater the 15-minute performance. Thus, every party was evaluating from a different point of view, which we hope to give some more perspective in interpreting the results with a changing performance such as in ITW.

At 24 months, passive ankle ROM had decreased in 10, remained the same in 4 , and increased in 15 children. No significant differences between the treatment groups were noted. This parallels with previous observations. ${ }^{3,9,12,13}$ The pathophysiology of ITW is still unknown and causal treatment is not available. Therefore, in ITW studies, both measurements and interventions have generally been directed toward increasing passive ankle dorsiflexion, expecting that the gait pattern changes following the change in structure. ${ }^{5}$ ITW participants have shown to have reduced flexibility and stiffer ankle joints compared with non-ITW peers. ${ }^{3,14}$ The persistent toe-toe gait is thought to contribute to the equinus often observed in older ITW children. ${ }^{15}$ Moreover, an early characteristic of ITW is the out-of-phase gastrocnemius activity before foot contact which can be modified with BTX-A. ${ }^{8}$ Brunt et al suggested that intervention should be directed toward both the neuromotor (functional exercise programs at home such as walking in heels, BTX-A, casts) and the musculoskeletal system (stretching, casts, splints). ${ }^{8}$

TWSS and ankle ROM are measures at the International classification of functioning, disability and health: children \& youth version (ICF-CY) level of body structures and function. ${ }^{11}$ TWSS is easy to use, but is subjective and yet nonvalidated. The scale designed and used in this study had interrater reliability varying between 0.30 and 0.70 (compared between independent rater and H. S.). Because of the reasons mentioned earlier, we sought to maximize reliability by having the same parent/researcher/blinded rater assess the children throughout the study. We also sought to evaluate the ICF-CY level of activity with the lower extremity function assessment test ${ }^{7}$ completed by the parents at pretreatment and each follow-up session. ITWchildren are generally considered high-functional, but they have difficulties in tasks requiring endurance, balance, and heel contact. The activities causing most difficulty for our patients included walking with heel strike, walking in heels, keeping the squat position with heels down, running with occasional heel contact, walking over $2 \mathrm{~km}$ distance, and tripping. Both groups improved during the 24-month follow-up. The most prominent increase in function was noted during the first 6 months, especially in the tasks mentioned. Our results parallel with those of Jacks et al. ${ }^{7}$

The conservative treatment methods in our study were chosen based on previous studies. ${ }^{6-10,12,13,15,16}$ We tried to make the interventions (firm shoes, night splints, stretching) as easy as possible to be integrated into the children's daily life. If the child had a sporty hobby, he/she was encouraged to continue and attach the stretching to that. The many options to integrate the stretching into the ordinary life were discussed with the research physiotherapist before and during the study period to keep up the motivation. Naturally, there were periods when forgetting to stretch (e.g., at summer vacations), which the parents admitted openly at assessments. Keeping a diary would probably have helped to be consistent. However, we did not require this because of thinking it to be too exhausting for a long time period of 2 years. Also, physiotherapy once a week was appreciated by the parents to help reminding of stretching on a daily basis. The night splints were well accepted and no skin or sleeping problems were noted. All children wore night splints and firm shoes regularly except one in $\mathrm{CO}$ group.

Toe-walking has been noted to resolve spontaneously in 12 to $79 \%$ of children. ${ }^{12,13,16}$ These retrospective natural history studies have included some form of intervention (physical therapy/stretching, firm shoes, night splints, bracing, or casting) and have studied children from 1.5 to 10 years of age with different outcome raters (parent or physician) and different follow-up from 2 to 21 years. A recent crosssectional study noted that, over $50 \%$ of children who started toe-walking early had ceased spontaneously at the age of 5.5 years. ${ }^{4}$ In our study, having started the interventions from 2 years onward, the effect of the natural resolving cannot be specifically defined because of not having a control group with observation only. Though general data on the long-term consequences (such as physical deformities or poor functional outcomes) are scarce or lacking, the data available advocates intervening ITW as early as possible. ${ }^{17}$ However, there are still no tools to predict who will resolve without intervention (i.e., with reassurance and observation), or who will benefit and from which treatment option. ${ }^{17}$

In conclusion, single or repeated BTX-A treatments did not significantly enhance the ITW-walking pattern compared with conservative treatment at 24 months follow-up. The 
ratings of parents were more modest compared with those of the blinded rater and research physiotherapist but may give a better indication of the child's situation in normal environment. Also, the difference in ratings highlights the challenges in measuring the degree of a changing walking pattern such as in ITW. The limitations of this study relate to the small sample size and risk of type II error of obtaining false-negative results. Thus, these results must be confirmed by studies with larger patient groups and more rigorous protocol of repeated BTX-injections during the follow-up period.

\section{Disclosures}

This study was supported, in part, by the Medical Research Funds of Tampere University Hospital, Kanta-Häme and Päijät-Häme Central Hospitals, and the Arvo and Lea Ylppö Foundation, Finland. The authors have no conflicts of interest directly relevant to the content of this article.

\section{Acknowledgments}

We thank the children and their families for participating in this study.

We also thank Tarja Pietikäinen, PT, and Terhi Iisalo, PT, Tampere University Hospital; Marja Salo, PT, Kanta-Häme Central Hospital; Merja Kervinen, PT, and nurse Virpi Schemeikka, Etelä-Karjala Central Hospital; Marja Korvenranta, PT, and Irma Tarvainen, PT, Päijät-Häme Central Hospital, Lahti, Finland; as well as Maarit Pekkala, PT, and Raija Lehtonen, PT, Oulu University Hospital.

\section{References}

1 Sutherland DH, Olshen R, Cooper L, Woo SL. The development of mature gait. J Bone Joint Surg Am 1980;62(3):336-353

2 Sala DA, Shulman LH, Kennedy RF, Grant AD, Chu ML. Idiopathic toe-walking: a review. Dev Med Child Neurol 1999;41(12): 846-848

3 Engelbert R, Gorter JW, Uiterwaal C, van de Putte E, Helders P. Idiopathic toe-walking in children, adolescents and young adults: a matter of local or generalised stiffness? BMC Musculoskelet Disord 2011;12:61

4 Engström P, Tedroff K. The prevalence and course of idiopathic toewalking in 5-year-old children. Pediatrics 2012;130(2):279-284

5 van Kuijk AA, Kosters R, Vugts M, Geurts AC. Treatment for idiopathic toe walking: a systematic review of the literature. J Rehabil Med 2014;46(10):945-957

6 Gormley ME, Herring GM, Gaebler-Spira D. The use of botulinum toxin in children: a retrospective study of adverse reactions and treatment of idiopathic toe-walking. Eur J Neurol 1997;4(Suppl 2): S27-S30

7 Jacks LK, Michels DM, Smith BP, Koman LA, Shilt J. Clinical usefulness of botulinum toxin in the lower extremity. Foot Ankle Clin 2004;9(2):339-348

8 Brunt D, Woo R, Kim HD, Ko MS, Senesac C, Li S. Effect of botulinum toxin type A on gait of children who are idiopathic toe-walkers. J Surg Orthop Adv 2004;13(3):149-155

9 Engström P, Gutierrez-Farewik EM, Bartonek A, Tedroff K, Orefelt C, Haglund-Åkerlind Y. Does botulinum toxin A improve the walking pattern in children with idiopathic toe-walking? J Child Orthop 2010;4(4):301-308

10 Engström P, Bartonek Å, Tedroff K, Orefelt C, Haglund-Åkerlind Y, Gutierrez-Farewik EM. Botulinum toxin A does not improve the results of cast treatment for idiopathic toe-walking: a randomized controlled trial. J Bone Joint Surg Am 2013;95(5):400-407

11 World Health Organization. International classification of functioning, disability and health: children \& youth version: ICF-CY. Geneva, Switzerland: WHO; 2001. Available at: http://apps. who.int/iris/bitstream/10665/43737/1/9789241547321_eng. pdf. Accessed March 24, 2016

12 Stricker SJ, Angulo JC. Idiopathic toe walking: a comparison of treatment methods. J Pediatr Orthop 1998;18(3):289-293

13 Hirsch G, Wagner B. The natural history of idiopathic toe-walking: a long-term follow-up of fourteen conservatively treated children. Acta Paediatr 2004;93(2):196-199

14 Williams C, Tinley PD, Curtin M, Nielsen S. Foot and ankle characteristics of children with an idiopathic toe-walking gait. J Am Podiatr Med Assoc 2013;103(5):374-379

15 Sobel E, Caselli MA, Velez Z. Effect of persistent toe walking on ankle equinus. Analysis of 60 idiopathic toe walkers. J Am Podiatr Med Assoc 1997;87(1):17-22

16 Eastwood DM, Menelaus MB, Dickens DR, Broughton NS, Cole WG. Idiopathic toe-walking: does treatment alter the natural history? J Pediatr Orthop B 2000;9(1):47-49

17 Oetgen ME, Peden S. Idiopathic toe walking. J Am Acad Orthop Surg 2012;20(5):292-300 